\title{
Digital Angioplasty Balloon Inflation Device for Interventional Cardiovascular Procedures
}

\author{
Xin $\mathrm{Ma}^{1}$, Zhong Fan ${ }^{2}$, Chee-Kong Chui* ${ }^{1}$, Yiyu Cai ${ }^{2}$, James H. Anderson ${ }^{3}$, \\ Wieslaw L. Nowinski ${ }^{1}$ \\ 1. Biomedical Lab, Kent Ridge Digital Labs, Singapore 119613 \\ 2. School of MPE, Nanyang Technological University, Singapore 639798 \\ 3. Johns Hopkins University School of Medicine, Maryland 21205, USA \\ Email: * cheekong@krdl.org.sq
}

\begin{abstract}
Percutaneous transluminal coronary angioplasty (PTCA) is an important interventional procedure performed everyday in major hospitals. In this paper, we describe our new designed angioplasty catheter balloon inflation device. It is a digital system that can be used in the actual PTCA procedure or other balloon angioplasty procedures. It is currently being used to simulate the inflation and deflation of angioplasty balloons in conjunction with an interventional radiology simulator. It also can be used in clinical procedures. The device has several outstanding advantages in addition to it resembling the existing handheld device used in the clinical setting. When used in the clinical procedures, a physician can manipulate the device using one hand with a remote controller as opposed to two-hand operating with the existing device. The manipulation accuracy is high allowing the physician to set the minimum inflation step at the level of 0.1 bar. Being an electromechanical device, it can be integrated better then the existing mechanical device, into the increasing digital clinical environment. New features such as intelligent programming can also be implemented using this new electromechanical device. This new angioplasty catheter balloon inflation device is integrated and validated with our interventional radiology simulator in the clinical setting.
\end{abstract}

\section{Introduction}

Since its introduction in the late 70's (1), percutaneous transluminal coronary angioplasty has become an important procedure that is performed daily in major hospitals. This technique consists of a catheter system introduced via a needle puncture in the femoral artery. A pre-shaped guiding catheter maneuvered, under realtime x-ray imaging into the orifice of the coronary artery. An angioplasty balloon catheter is then advanced through this guiding catheter selectively into the stenotic segment of the coronary artery. After traversing the stenotic lesion, the balloon is inflated by a pump-controlled pressure. This pressure compresses the arteriosclerotic material in a direction perpendicular to the wall of the vessel and thereby dilating the vessel lumen. The pressure injector that is used to inflate the balloon is also known as the balloon inflation device. The first balloon inflation device is developed by Dr. Andreas Gruentzig [1]. It is an important component of the PTCA and other related 
procedures such as stent placement. Commercially available devices are based on the original mechanical design of the Gruentzig's device.

Physicians have to be careful in performing balloon angioplasty procedures so as to avoid serious injury, such as rupturing or dissecting the wall of the vessel. There are normally two ways to reduce potential injury. One is to provide a better training environment for physicians to acquire the necessary skill levels and experience to avoid injury. Although practicing on live patients can provide excellent training, there exist a limited number of cases and alternative training methods are needed. A realistic vascular catheterization simulation system could provide the type of training the physicians desires [2][3]. The alternative is to improve the performance of medical devices used in the procedures so that they can be used easily by the physician, and are safe to patients.

The balloon injection device currently used in clinical procedures cannot provide the electric signals that are needed for our simulation system. We therefore designed a digital handheld balloon injection device that is an integral component of our vascular catheterization simulator. A challenge is to provide a simulated device that closely resembles the existing device in the clinical setting. It should be able to response by delivering the correct signals corresponding to user's manipulation.

As an additional result, we designed an automatic version of the handheld balloon injection device that can be used not only as a component of our simulation system, but also in real clinical angioplasty procedures. When used in the clinical procedures, physician may use a handheld remote controller to actuate the inflation/deflation procedure allowing for one hand operation. The manipulation accuracy is high allowing physicians to set the minimum inflation steps in the level of 0.1 bar. Finally physicians can operate the device work in an "intelligent" way by setting alarms. For example, physicians can set the rate of balloon inflation alarm and maximum pressure alarm according to their experience and according to manufacture specifications. During the procedure, the micro-controller inside the device can calculate the rate and pressure of inflation. If the value is over the alarm value, a buzzer will alarm the physician. This provides a safety factor to reduce potential balloon overinflation, rupture and serious clinical complications.

In the remaining sections, we will first describe the design and mechanism of our balloon inflation devices (handheld one and automatic one), and then discuss the results from integration with the interventional radiology simulator.

\section{Handheld Catheter Balloon Inflation Device}

In this section, we will describe the catheter inflation device for simulating balloon inflation and deflation with the interventional radiology simulator. This device is handheld and closely resembles the actual device used in the clinical setting. .

The handheld catheter based balloon inflation device was made for realistic training simulation. It has all the essential components of an existing balloon inflation device, and is different from these currently available handheld syringe pump device by the addition of a digital pressure switch with analog output for PC data interface. Also instead of the standard inflation medium (an X-ray radiopaque 
contrast based mixture), air is used. In actual clinical setting, air is not injected for fear of balloon rupture and air embolism that could be lethal.

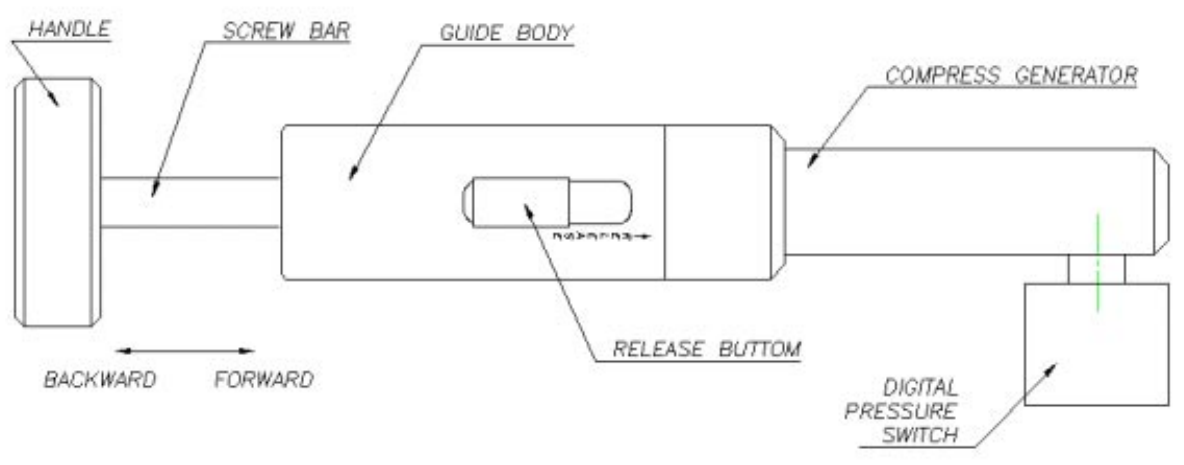

Figure 1. Drawing of handheld digital balloon inflation device

The specifications of the device are listed below:

\begin{tabular}{l|l}
\hline \multicolumn{1}{c|}{ Specification } & \multicolumn{1}{c}{ Value } \\
\hline Maximum liquid pressure & 20 Bar (2 Mpa, 20 ATM) \\
Compress stroke & Around 2 inches \\
Pressure switch & Analog output: 1 to 5V \\
\hline
\end{tabular}

This device can provide up to 20 Bar of compressed liquid by means of a manual screw and nut mechanism. The user can twist the handle (clockwise) and the screw bar forces the piston (inside the compressed liquid generator) forward to compress the liquid which is sealed in the compress generator. The release button can be used to release the compress liquid quickly (balloon deflation).

The digital pressure switch measures the balloon inner pressure directly, and allows one to control the intra-balloon pressure as indicated by the digital LED display more easily. The existed handheld inflation syringe has markers that are used to determine the balloon pressure, but it has more tolerance for actual pressure measurement. Meantime, the digital pressure has analog output and PC can collect this signal to simulate the procedure of inflating/deflating balloon. In addition to the pressure, we are track the rate of inflation and deflation.

\section{Automatic Catheter Balloon Inflation Device}

The existing handheld balloon inflation device must be held by one hand and twisted by the other hand, during the procedure. One hand operation of the balloon inflation provides the physician cardiologist with an easier means to initiate and monitor balloon inflation. Accordingly, we designed a desktop-based automatic catheter 
balloon inflation device on the base of our handheld one. It can be used not only with our interventional radiology simulator but also in real clinical procedures.

To convert the manual handheld device to an automatic model, we utilize an AC linear motor for contrast liquid compression instead of the screw and nut mechanism. This provides an automatic liquid compression actuation. The compress liquid generator and digital pressure switch is the same as for the handheld system. The pressure output connects the balloon catheter for actual operation use while it is implemented for simulation proposes. All of the components of the above unit are integrated as one desktop device. An additional handheld remote control device with a digital pressure display has push buttons that are design for manual mode operation and for fine pressure adjustments. The micro-controller (programming controller) is a core unit for electromechanical interaction with other units. In clinical procedures, this device is an embedded system. The simulation PC and network/internet interface are additional components to the device. In simulating procedure, the simulator PC is connected and used to observe and verify the operations of a pressure data processing system and indicates any significant departure from the norm. Alternately, the PC can record data from the practice operation for study and simulation databases. The network/internet interface is intended to connect with other devices, such as respirators and blood pressure monitoring systems utilized in the clinical environment. The system can also be used for remote training and telemedicine. The design of this device is shown in the following schematic block diagram (Figure 2).

The device can work in three modes: manual mode, auto mode and simulation mode. The manual and auto modes allow user to use the device in actual clinical setting. The device has been used in conjunction with our interventional radiology simulator in simulation mode. Nevertheless, it can be used with other simulation systems on PC platform.

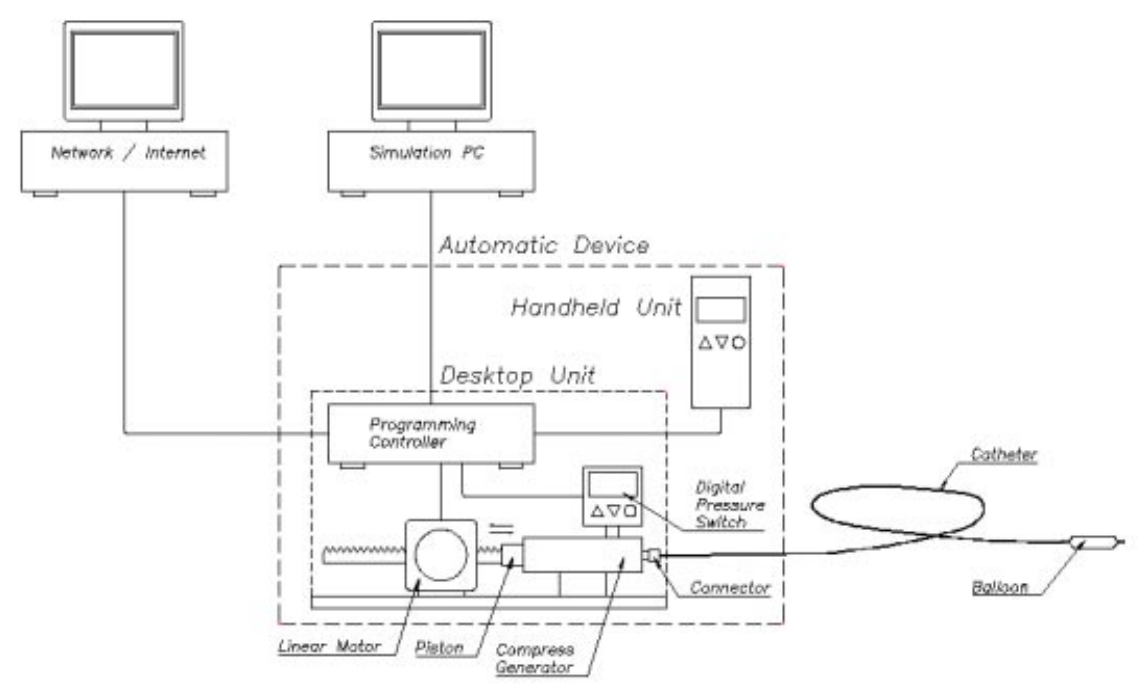

Figure 2. Block diagram of automatic digital balloon inflation device 


\section{Manual Mode}

In manual mode, the user first selects the maximum pressure to be delivered. This depends on the specific type of catheter balloon device used. The user then starts to increase the pressure by means of a button on the handheld remote control and monitors the pressure display. The monitor PC will check the rate of balloon pressure increase that was pre-selected based on the specific catheter balloon device chosen. If an incorrect action occurs, a buzzer will alarm the user. The system automatically maintains the proper pressure within the balloon during the procedure.

\section{Auto Mode}

When in auto mode, the user first selects the type of catheter balloon device to be used. This is done from a library of clinically available catheter balloon device specifications stored in the PC. The user then keys in the maximum pressure as a check. The pre-programming operation will begin after pressing the start button. The user can still monitor the pressure data through the handheld remote control and can stop the programming by means of a stop button located on the remote control. The monitor PC will analyze the data from network I/O to automatically adjust the pressure and increase/decrease speed accordingly.

These two allow the physician to operate the device work more intelligently. Physician can set some alarm values such as balloon inflation rate and maximum inflation pressure alarm according to their clinical experience. If alarm is generated during the clinical procedure, proper steps can be taken by the physician to avoid balloon overexpansion or rupture with subsequentand injury to the patient.

\section{Simulation Mode}

In a clinical procedure, the device works as an embedded desktop system. All the information and control signals are processed and generated by the micro-controller inside as shown in Figure 2. For simulation purposes, the device can be connected to a PC. The device can be used as a component of an interventional radiology simulator. The PC also can be used to record data during actual angioplasty procedures. The simulation environmenet is very useful for pretretment planning or training.

The automatic catheter balloon inflation device has three advantages. The first is that physician can complete the whole balloon inflation procedure using one hand. The second relates to the high accuracy of the system in monitoring and displaying inflation pressures. The minimum inflation step is 0.1 bar. The third is that with an intelligent alarm setting, the procedure can prevent balloon overinflation and potential clinical complications. 


\section{Results}

We have integrated the handheld balloon inflation device into our interventional radiology simulator. The pressure switch of the balloon inflation device can apply an analog voltage output in the range of $1-5 \mathrm{~V}$. With this analog signal, the device is now used as a component in our TiC (Tactile and Image Control) interfacing system [4]. The analog signal is firstly alternated into digital signal and then inputted into a PC through a serial port. The signal is then used to simulate inflation/deflation of a balloon catheter to treat the stenotic lesion in the virtual blood vessel.

Figure 3 (a) shows the handheld balloon inflation device for simulation purposes. Figure 3(b) illustrates the simulation process using the device. Figures 4 and 5 are snapshots of the graphic display from our interventional radiology simulator [5]. Figure 4, illustrates inflation of the angioplasty in the human cerebral vasculature. Figure 5 shows a stent deployed by balloon inflation at the middle portion of the same cerebral artery. This provides a life-like simulation environment for the user to practice or for pretreatment planning of pertaneous transluminal coronary angioplasty procedures.

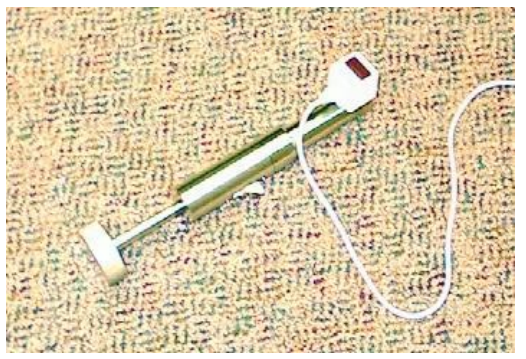

(a)

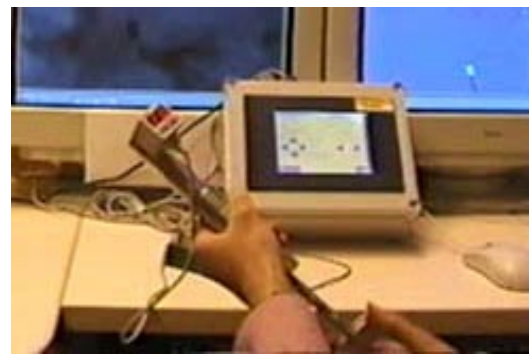

(b)

Figure 3 (a) Balloon inflation device. (b) Simulating balloon inflation and deflation

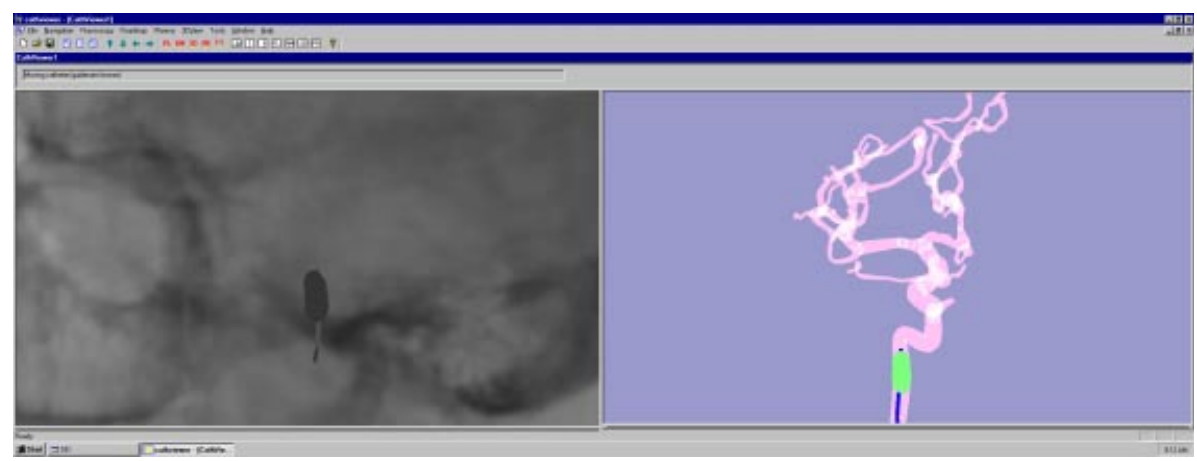

Figure 4. Simulated balloon inflation (Fluoroscopic and 3D views). 


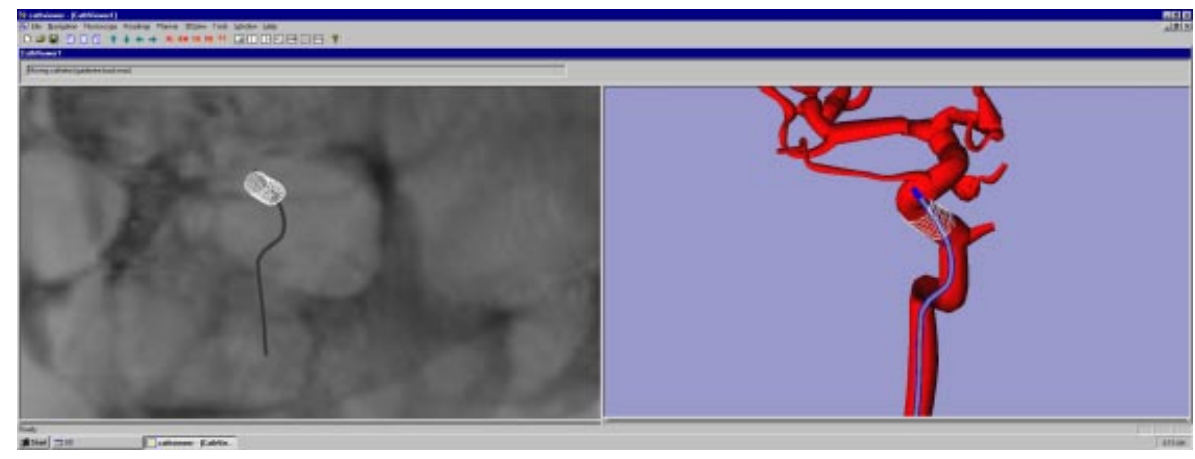

Figure 5. Simulated stent deployment with balloon inflation (Fluoroscopic and 3D views).

\section{Conclusion}

The catheter balloon inflation device described here, has been successfully used as a component of our interventional radiology simulator. Compared with normally used balloon inflation devices, it provides an electronic output signal that is useful for simulation purposes. We also designed a new desktop-based automatic catheter balloon inflation device that can be used not only in the simulation system, but also in clinical procedures. The manipulation of the device allows the physician to complete the balloon inflation procedure using one hand. The accuracy of the system in recording and displaying inflation pressures is very high. Finally, physicians can control the clinical procedure according to the pressure display on the handheld remote controller and be provided with additional balloon inflation safety alarm features.

Acknowledgement: Support of this research development by National Science and Technology Board of Singapore is gratefully acknowledged. We are grateful to Dr. Anthony C. Venbrux and Dr. Kieran Murphy of Johns Hopkins Hospital, Baltimore, USA for championing the validation effort in the clinical setting.

\section{Reference}

[1]. L.A. Geddes and L.E. Geddes, The Catheter Introducer, Mobium Press, Chicago, 1997. [2]. J. Anderson, W. Brody, C. Kriz, Y.P. Wang, C.K. Chui, Y.Y. Cai, R. Viswanathan and R. Raghavan, daVinci- a vascular catheterization and interventional radiology-based training and patient pretreatment planning simulator, Proc. of Society of Cardiovascular and Interventional Radiology (SCVIR) $21^{\text {st }}$ Annual Meeting, Seattle, USA, March 1996.

[3]. Y. Wang, C.K. Chui, H.L. Lim et al, Real-time interactive simulator for percutaneous coronary revascularization procedures. Computer Aided Surgery, Vol 3, No 5, 211-227, 1998. 
[4]. C.K. Chui, P. Chen, Y. Wang et al, Tactile Controlling and Image Manipulation Apparatus for Computer Simulation of Image Guided Surgery, Recent Advances in Mechatronics, Springe-Verlag, 1999.

[5]. C.K. Chui, J. Anderson, W. Brody, W.L. Nowinski, Report on Interventional Radiology Simulation Development, Internal Research Report, KRDL-JHU 2001. 